\title{
LA FILOSOFÍA PRÁCTICA EN LEIBNIZ: NIVELES DE ANÁLISIS, TENSIONES Y CONVERGENCIAS
}

\author{
Maximiliano Escobar Viré \\ Universidad Nacional de Quilmes
}

\begin{abstract}
RESUMEN: Este trabajo intenta formular un aporte crítico-hermenéutico para la reconstrucción de la ética y del pensamiento jurídico-político de Leibniz. Dicho aporte se centra en el concepto de filosofía práctica, que puede entenderse de dos modos: como un estudio del sumo bien y del modo de orientar la acción humana hacia éste, pero también como una actividad particularmente comprometida con la realización de ese bien. En este sentido, se sostendrá que la reconstrucción de la filosofía práctica de Leibniz requiere atender a estos dos niveles: el propio de una disciplina filosófica, y el de la dimensión práctica que reviste la filosofía per se, por ser la disciplina encargada de confirmar la contingencia del mundo, la libertad humana y la justicia de Dios. Estos dos niveles coexisten tensamente con una dimensión pragmática del filosofar, necesaria para alcanzar acuerdos políticoinstitucionales demandados por el contexto de la Europa de los siglos XVII-XVIII.
\end{abstract}

PALABRAS CLAVE: Filosofía práctica; justicia; conciliación; verdadera piedad.

\begin{abstract}
This paper intends to propose a critical and interpretative contribution to reconstruct Leibniz's ethics and political thought. That contribution focuses on the concept of practical philosophy, which may be understood in two different ways: as a study of the greatest good and the way to direct human action towards it, but also as an activity particularly engaged in the attainment of that good. Accordingly, this paper will hold that the reconstruction of Leibnizian practical philosopby requires a careful attention to these two levels: that of a philosophical discipline and that of the practical dimension involved in philosophy per se, in virtue of being the discipline which must confirm the world's contingency, human freedom and God's justice. Both these levels coexist in tension with a pragmatic dimension of philosophizing, which was required to reach political and institutional agreements largely sought in the European context of the seventeenth-eighteenth centuries.
\end{abstract}

KEYWORDS: Practical philosophy; justice; conciliation; true piety.

La reconstrucción de la ética y del pensamiento jurídico-político de Leibniz parece constituir uno de los desafíos que más interés viene despertando en el campo de los estudios leibizianos en las últimas décadas. Parte de ese interés tiene que ver con tratar de esclarecer la vinculación entre las ideas 
leibnizianas y los diversos proyectos de orden práctico que emprendió en vida, entre los cuales se destaca su prolongada búsqueda de unión entre las iglesias cristianas. La persistente labor negociadora que Leibniz asumió en tal empresa parece compensar la poca originalidad que suele atribuirse a su pensamiento en los ámbitos de la ética y de la política. Ese esfuerzo conciliador, intelectual a la vez que diplomático, parece alentar secretamente nuestras esperanzas por llegar a vislumbrar, entre sus avatares, las claves de un pensamiento en acción.

Este trabajo se propone formular un aporte crítico-hermenéutico para la comprensión y la reconstrucción de la filosofía práctica de Leibniz. Sin embargo, como se intentará sostener aquí, la idea de una filosofía práctica adquiere, en Leibniz, una significación que excede los límites de una mera rama disciplinar, y se proyecta hasta abarcar casi la totalidad de $s u$ filosofía. En la reconstrucción que aquí se esbozará, la racionalidad práctica parece estar atravesada, en Leibniz, por una tensión entre las exigencias de una justicia universal y la necesidad de adoptar una actitud pragmática para allanar la solución de los conflictos políticos y religiosos. Sin embargo, la comprensión de esta significación más amplia del carácter práctico de la filosofía nos permitiría captar, con mayor nitidez, un perfil no siempre atendido del pensamiento leibniziano. Desde tal perspectiva, la superación de las divisiones y la promoción del bien general se erigen como motor y espíritu del filosofar. 


\section{Dossiê Leibniz, Dissertatio - Volume Suplementar 03 | UFPel [2016]}

\section{La parte práctica de la filosofía}

De su maestro, Jacob Thomasius, Leibniz tomó desde joven una concepción de proveniencia aristotélica en torno a la filosofía práctica, concebida como una de las divisiones tradicionales de la filosofía. Tal concepción puede reconstruirse a partir de las anotaciones que formuló al manual de Thomasius (titulado precisamente "Philosophia practica"). La disciplina general así denominada incluía como primera parte (priorpars) a la ética, que era la doctrina que se ocupaba del fin último o del sumo bien de los hombres. ${ }^{1}$ Consecuentemente con el modelo aristotélico, la segunda parte (posterior pars) era la política, la cual se ocupaba del sumo bien civil en cuanto tal (subjecto), que es la ciudad (Civitas), así como del medio para alcanzarlo, que son las leyes. ${ }^{2}$ En la conocida carta a Thomasius de abril de 1669, Leibniz escribe a su maestro que de él aprendió que esta disciplina general, en sus dos divisiones, es una y la misma ciencia. ${ }^{3}$ Sin embargo, ya en esa carta, Leibniz denomina a la disciplina general como "filosofía moral", mientras que su primera división (la ética) es referida como la parte "práctica", por oposición a la parte "civil". ${ }^{4}$

Se ha sostenido que, en el siglo XVII, la ética de Aristóteles constituía la parte menos cuestionada de la filosofía aristotélica. En todo caso, su oposición principal provenía del rescate de las concepciones antiguas no-aristotélicas,

\footnotetext{
${ }^{1}$ AA VI 1, p.42.

${ }^{2} \mathrm{AA}$ VI 1, p.63.

${ }^{3} \mathrm{AA}$ II 1, p.20; GP I, p.22.

${ }^{4}$ A pesar de esta divergencia terminológica, entiendo que la fuente de referencia para aprehender la concepción leibniziana se encuentra en el manual de Thomasius.
} 
como el platonismo, el epicureísmo, el estoicismo y el escepticismo. Este rescate se había iniciado en el Renacimiento y, en general, era formulado en versiones no muy conflictivas con una visión cristiana. ${ }^{5}$ En cualquier caso, la parte práctica de la filosofía era esencialmente teleológica, eudemonista, y su tarea fundamental era la clarificación del bien supremo del individuo y de la comunidad política, así como de las virtudes que permitían alcanzarlos.

La parte de la ética que Leibniz heredó no sólo contenía una reflexión sobre el bien y una clasificación de las virtudes, sino que además incluía el tratamiento de la justicia universal y del derecho (Iure). En este punto, y en la subsiguiente sección de la política, las influencias no son sólo los autores clásicos (como Cicerón), sino que aparecen también los teóricos del iusnaturalismo moderno: Grocio, Hobbes y Pufendorf. Esto no sólo da cuenta de la pluralidad de fuentes de la que emerge la concepción leibniziana de la filosofía práctica, sino que revela también la presencia de lo que constituiría el principal foco de interés de Leibniz en esta disciplina: el tema de la justicia universal como fundamento para desarrollar una teoría del derecho que, desde el paradigma metodológico de los autores modernos, sea conciliable con la religión cristiana y con la teología natural.

El interés leibniziano por la ciencia del derecho o jurisprudencia se explica, en parte, por su formación profesional como jurista, carrera a la que habría de dedicarse por el resto de su vida. Además, la cuestión del derecho natural

${ }^{5}$ Kraye (2008). 


\section{Dossiê Leibniz, Dissertatio - Volume Suplementar 03 I UFPel [2016]}

se hallaba en el epicentro del debate, a raíz de la irrupción del iusnaturalismo moderno. Esta orientación de su pensamiento se vio impulsada por su pronta llegada a la Corte de Mainz, a sus 21 años (1668), donde comenzó a colaborar en el proyecto para ordenar y armonizar el Corpus legal. Esta tarea condujo a Leibniz a elaborar los escritos reunidos bajo el título de Elementa juris naturalis (1669-1671), que constituyen la primera versión de lo que sería su proyecto en el campo del derecho: formular una jurisprudencia universal, concebida como una ciencia demostrativa del derecho, basada en la noción de justicia y construida a partir de una ordenación y una racionalización del derecho positivo romano. Tal racionalización debía mostrar la coincidencia fundamental entre el derecho romano y el derecho natural. ${ }^{6}$

Sin embargo, el interés de Leibniz por la teoría jurídica no provenía sólo de su profesión, sino también de una vocación muy personal. Esa vocación puede descubrirse en la orientación general que adquiere su filosofía ya desde los escritos de juventud, que es claramente apologética y se dirige a mostrar la convergencia de la razón con la fe cristiana. Por ello, lo que distinguirá a la doctrina leibniziana del derecho natural es que ella supone, y exige demostrar, las verdades fundamentales de la teología natural: la existencia de Dios y la inmortalidad del alma. Así lo afirma Leibniz ya en su Nova Methodus discendae docendaeque Jurisprudentiae, de 1667.7 Si no se dieran tales verdades, los preceptos

\footnotetext{
${ }^{6}$ Artosi, Pietri y Sartor (2013); xix, xx; Rateau (2008), pp.78-79. Rateau destaca más bien el carácter "polifónico" de los escritos jurídicos leibnizianos de juventud, en los que habría un hilo conductor, que sería la búsqueda de una unidad sistemática del derecho natural, subyacente al derecho positivo romano.

${ }^{7}$ AA VI 1, p.344-345.
} 
del derecho natural carecerían de todo "vínculo físico" con el mundo, ya que no habría un garante de su ejecución, dispensador de beatitud y condenación en una vida futura. ${ }^{8}$ Ello muestra, a su vez, la necesidad de probar que Dios es justo, y que la noción de justicia es común a Dios y a las criaturas en tanto espíritus racionales.

Esta justicia universal será el objeto primordial de la jurisprudencia leibniziana, "ciencia de las acciones en tanto son llamadas justas o injustas". $9 \mathrm{Su}$ clarificación constituirá el paso obligado para la comprensión de sus divisiones específicas: la justicia divina (referida al mundo y a su gobernador), la bumana (referida al género humano) y la civil (propia de la república). ${ }^{10}$ Esta tarea requiere del filósofo, ya que la regla suprema y los preceptos del derecho se derivan, en definitiva, de la misma noción de justicia, la cual sólo puede ser concebida por el entendimiento. En efecto, la regla suprema del derecho manda "encaminar todos nuestros actos a la consecución del bien general”. De ella se siguen los tres preceptos del derecho: "vivir honestamente" (precepto de la justicia universal), "no hacer daño a nadie" (precepto de la justicia conmutativa, base del derecho privado) y

${ }^{8}$ AA VI 1, p.344. Cf. también AA II 1, p.30; AA VI 1, p.437. Esto conlleva el rechazo a la aspiración de Grocio a formular una teoría del derecho natural que preservaría su validez aún si concediésemos que Dios no existe ("etiamsi daremus (...) non ese Deum"). Cf. De iure belli ac pacis, "Prolegomena", § 11. ${ }_{9}^{A} \mathrm{AA} V I 11$, p.300. Grua recuerda que en los siglos XVI y XVII, el derecho común a las personas de diversos países se denominaba "derecho natural" o "derecho de gentes", según se fundara en la naturaleza o en el uso. Este derecho es referido por algunos autores como "jurisprudencia universal". Cf. Grua 1953: 158. Esta expresión, sin embargo, adquiere en Leibniz la significación de una doctrina de la justicia universal, común a Dios y a las criaturas y base del derecho completo.

${ }^{10} \mathrm{AA}$ VI 1, p.300-301. En la misma obra, el Nova methodus, Leibniz señala que la teología es parte de la jurisprudencia universal, en tanto esta comprende leyes tanto humanas como divinas. Tal jurisprudencia tiene un doble principio: en parte la razón, y de aquí la teología y la jurisprudencia naturales (lo que incluye las obras de Hobbes y Grocio), y en parte la escritura. La teología es una especie cuyo universal es la jurisprudencia. Cf. AA VI 1, p.294. Cf. también Rateau (2008), p. 70-72. 


\section{Dossiê Leibniz, Dissertatio - Volume Suplementar 03 UFPel [2016]}

"dar a cada uno lo que le corresponde de acuerdo al bien de la sociedad en general" (precepto de la justicia distributiva, base del derecbo público). ${ }^{11}$

Así, la filosofía práctica parece constituir, para Leibniz, una ciencia racional, cuyo tema central son las verdades eternas basadas en la definición de la noción común de justicia. ${ }^{12}$ Tales verdades fundan las leyes de un orden moral universal, un reino de la gracia encaminado hacia el bien común (la gloria de Dios), al cual debieran orientarse también las repúblicas del mundo humano.

Resta señalar que la expresión "filosofía práctica", en tanto expresión empleada para referir a la parte práctica de la filosofía, tiende a desaparecer en el vocabulario leibniziano de madurez. ${ }^{13}$ La expresión más habitual en sus escritos, y la que Leibniz parece preferir, es "filosofía moral".

\footnotetext{
${ }^{11}$ Se trata de los tres preceptos atribuidos a Ulpiano (siglos II-III d.C.) y recogidos en el Corpus juris civilis de Justiniano. Tales preceptos constituirán, para Leibniz, el núcleo racional del derecho natural, presente ya en el derecho romano. Cf. Salas 111-112 ("Sobre los tres grados del derecho natural y el de gentes", Mollat 8-9).

${ }_{12} \mathrm{AA} \mathrm{VI}, \mathrm{i}, \mathrm{p} .460-461$.

${ }^{13}$ La expresión reaparece en la correspondencia con Wolf, en boca de este último, quien escribe que en su "filosofía práctica", el bien privado, el bien público y la gloria de Dios se distinguen como el fin y los medios. Cf. LW 36. Asimismo, en la carta a Thomas Burnett del 1/11 de febrero de 1697, Leibniz distingue la filosofía teórica respecto de la filosofía práctica sosteniendo que la primera se funda sobre el análisis de las nociones, mientras que la segunda se funda "sobre la verdadera Tópica o Dialéctica, es decir, sobre el arte de estimar los grados de las pruebas, que no se encuentra incluso en los autores lógicos, pero del cual sólo los jurisconsultos han dado muestras que no se deben menospreciar" (GP III, p.193). En este último sentido, la filosofía práctica se distingue de la teórica por el tipo de metodología requerida más bien que por el objeto de estudio: se trata de una disciplina en la cual se requiere verificar hechos históricos o interpretar el sentido de los textos, y en la cual sólo puede alcanzarse una certeza moral. El contexto de esta distinción es el de esclarecer si es posible tratar los temas de la teología "methodo mathematica" (GP III, p.190). La postura general de Leibniz es que esto es posible, aunque requiere no sólo del análisis de las nociones, sino también de la interpretación del texto sagrado, así como de la verificación histórica de su autenticidad. Estas tareas requieren del "arte de estimar los grados de las pruebas", al cual Leibniz se refiere aquí en términos de "filosofía práctica".
} 


\section{La dimensión práctica de la filosofía}

Sin embargo, en los escritos de Leibniz puede descubrirse una concepción más profunda, según la cual toda su filosofía tendría una significación práctica.

En algunas de sus cartas, Leibniz construye un relato del amanecer de su vocación filosófica en la infancia y la primera adolescencia. Según esta escenificación, a sus quince años se encontró con dos obras que lo afectaron vivamente: el De servo arbitrio, de Lutero, y el De libero arbitrio, de Lorenzo Valla. Ambas obras lo habrían impulsado a asumir la difícil tarea de conciliar la predestinación divina con la libertad humana, como si se tratara de una misión encomendada por Dios mismo. ${ }^{14}$ La reiteración constante de esta anécdota parece erigirse como una llave maestra para acceder al corazón mismo de la filosofía leibniziana, al núcleo motivacional que establece su cauce. El mismo Leibniz evoca esta escena como el momento de iniciación de la reflexión que conduciría nada menos que a sua Théodicée de madurez. ${ }^{15}$

Para Leibniz, este problema no constituía un desafío aislado, sino que se insertaba, más bien, en un conjunto de aporías que amenazaban la consistencia lógica y la viabilidad racional de toda defensa filosófica del Cristianismo. ${ }^{16}$ Por ello, los escritos y las cartas de Leibniz, a partir de 1668,

\footnotetext{
${ }^{14} \mathrm{AA} 118$, p.322 ( $\left.\mathrm{N}^{\circ} 194\right)$, carta a Daniel Ernst Jablonski del 23 de enero (2 de febrero) de 1700. El mismo relato, con algunas diferencias, puede encontrarse en: GP III 481 (a Jaquelot, 1704-1705); GP III, p.143 (a Basnage, 19 de febrero de 1706); Grua 459 (1705); 497 (1707). ${ }^{15} \mathrm{GP}$ VI, p.43.

${ }^{16} \mathrm{AA}$ VI 3, p.1117, la Confessio philosophi de 1672-1673.
} 


\section{Dossiê Leibniz, Dissertatio - Volume Suplementar 03 I UFPel [2016]}

revelan la aspiración a construir una "filosofía verdadera", capaz de demostrar las creencias básicas de la religión cristiana, como la existencia de Dios y la inmortalidad del alma. ${ }^{17}$ Estas constituyen, para él, un fundamento necesario tanto para la jurisprudencia universal como para la explicación mecanicista de los fenómenos físicos. ${ }^{18}$

Sin embargo, lo que a Leibniz más interesaba es que esta filosofía verdadera estableciera sobre bases sólidas una convicción moral, centrada en la piedad cristiana. Como escribe a Thomasius en 1669, dicha filosofía debe ser "una balsa" para los piadosos, contra la amenaza del ateísmo (que Leibniz vislumbraba en el mecanicismo exacerbado de los pensadores modernos). $\cdot{ }^{19}$ Para ello, Leibniz consideraba prioritario resolver el problema que oponía la predestinación a la contingencia y a la libertad humana, como si se tratara de doctrinas incompatibles. Más aun, esta respuesta tenía, a sus ojos, una urgencia y una significación de orden práctico. Así lo revelan escritos de diversas épocas, en los que Leibniz dice claramente que esta cuestión es la que acarrea más riesgo, ${ }^{20}$ y que tiene una gran importancia en relación a la conducta en la vida. ${ }^{21}$ Esta convicción llega hasta la Théodicée, en los que Leibniz recuerda que hay dos laberintos para la mente humana: aquel de la composición del continuo, y "la

\footnotetext{
${ }^{17} \mathrm{Cf}$. AA VI 2, p.395. En una carta a Johann Friedrich de Hannover, Leibniz se refiere a los "Elementos demostrados de la verdadera filosofía", que debían preceder a su proyecto de las Demostraciones católicas ya en 1669. Cf. AA II 1, p.489; AA VI 1, p.494.

${ }^{18}$ Confessio naturae contra atheistas, AA VI 1, p.489-490 y ss. Cf. también AA II 1, p.24; AA VI 1, p.513.

${ }^{19}$ AA II 1, p.24; cf. también AA VI 1, p.513.

${ }^{20}$ AA VI 1, p.537.

${ }^{21}$ AA VI 4, p.1406.
} 
gran cuestión de lo libre y lo necesario". Allí afirma también que, mientras el primer asunto incumbe sólo a la especulación (teórica), el segundo es importante "para la práctica". 22

¿Cómo se puede interpretar esta viva preocupación leibniziana?

Contra todo escepticismo, Leibniz no duda en que la filosofía establece la verdad. Como afirma en su madurez, la rąón misma no es otra cosa que "el encadenamiento de las verdades". ${ }^{23}$ Y la verdad es lo que decide, en definitiva, cómo debemos comportarnos en esta vida, ya que ella determina si hay Dios, si el alma es inmortal, y si podemos esperar algún gozo por nuestra conducta virtuosa, o si los injustos no recibirán castigo alguno por el mal infligido. De allí la importancia de demostrar que las criaturas racionales poseen una verdadera libertad, concebida no sólo como ausencia de necesidad absoluta, sino también como la espontaneidad racional que vuelve a cada espíritu la verdadera fuente de sus acciones. ${ }^{24}$ En este sentido, la filosofía teórica adquiere, por sí misma, una dimensión práctica, ya que el conocimiento de la verdad establece las bases de la piedad.

Por ello, a lo largo de su vida, Leibniz encaró su filosofía como una lucha contra las pretensiones "naturalistas" del mecanicismo moderno, que en sus versiones epicúrea o estoica intentaban explicar el mundo sin recurrir a Dios

${ }^{22}$ Cf. GP VI, p.29.

${ }^{23} \mathrm{GP}$ VI, p.49; Salas, p.411.

${ }^{24}$ AA VI 4 1653; GP VI 385. 


\section{Dossiê Leibniz, Dissertatio - Volume Suplementar 03 I UFPel [2016]}

como ratio ultima; ${ }^{25}$ también contra el ateísmo que asomaba en quienes reducían la providencia divina a una "necesidad ciega", como Hobbes y Spinoza, y contra aquellos que tergiversaban o lanzaban dudas escépticas contra los fundamentos racionales de la verdadera religión, como los socinianos y Bayle respectivamente. Frente a todas estas doctrinas, Leibniz sostuvo que la filosofía debía brindar una noción de Dios y de su perfección que pudiera servir en la física y en la moral, mostrando que "la causa eficiente de las cosas es inteligente, tiene una voluntad, y por tanto tiende al bien". ${ }^{26}$

La dimensión práctica de la filosofía (teórica) no sólo estriba en que confirma la verdad de la religión. Además, ella conduce al espíritu hacia la piedad y la virtud. En sus escritos sobre la felicidad y la sabiduría, ${ }^{27}$ Leibniz consolida una concepción ética intelectualista, en la cual el mayor placer y el sumo bien del espíritu sólo pueden residir en el conocimiento del ser más perfecto. Y no podríamos amar a Dios sin conocer sus perfecciones, ya sea a través del conocimiento de las verdades eternas o del de la armonía del universo. ${ }^{28}$ El conocimiento de Dios nos lleva a amarlo, y el auténtico amor a Dios nos lleva a imitarlo, es decir, a buscar el bien común. ${ }^{29}$ En este sentido, es claro que para Leibniz, la filosofía (concebida como la búsqueda de un conocimiento distinto

\footnotetext{
${ }^{25}$ La distinción entre las dos "sectas" de los nuevos "naturalistas", que se basan en Epicuro o en los estoicos, se encuentra formulada en un escrito de 1678-1680. Cf. AA VI 4 1384-1386.

26 GP IV 284.

27 Grua $579-588$.

28 Grua 580-581.

${ }^{29}$ Grua 586-587.
} 
sobre la última razón de las cosas) opera una preparación o transformación del espíritu hacia la suprema virtud... que sólo puede ser la suprema piedad.

\section{La dimensión pragmática de la filosofía}

La "verdadera filosofía" proyectada por Leibniz tenía una dimensión práctica no sólo porque aportaba bases sólidas para confirmar la fe de los piadosos. Además, ella resultaba indispensable, a sus ojos, para alcanzar un fin político-institucional fuertemente anhelado en la Alemania de mediados del siglo XVII: la restauración de la unidad entre las iglesias cristianas, divididas desde la Reforma.

Desde su arribo a Mainz, Leibniz adhirió a la tendencia irénica de los calixtinos que propiciaba esta reunión. ${ }^{30}$ A lo largo de su vida, participó en varias negociaciones para acercar a las facciones protestantes entre sí (luteranos o “evangélicos" por un lado, calvinistas o "reformados" por el otro), y a la Iglesia Romana con las confesiones protestantes. Los puntos de divergencia eran difíciles de resolver:

1. en el plano teórico, había profundas diferencias en a) la interpretación de los misterios (la trinidad, la encarnación, la eucaristía, la resurrección de los cuerpos), b) el problema de la predestinación y de la libertad, con sus asuntos concomitantes (como el concurso divino y el origen del mal) y c) algunas doctrinas menos centrales, como la legitimidad del divorcio;

\footnotetext{
30 de Olaso 77. Los "calixtinos" derivaban su nombre de Georg Calixtus (1585-1656), teólogo luterano que había promovido el movimiento reunificador en la primera mitad del siglo XVII. En Mainz, a la llegada de Leibniz, el movimiento era conducido por su hijo Frederic Ulric y por Hermann Conring, jurista con quien Leibniz mantendría un diálogo epistolar.
} 


\section{Dossiê Leibniz, Dissertatio - Volume Suplementar 03 I UFPel [2016]}

2. en el plano práctico, se trataba de resolver cuestiones vinculadas a la autoridad y el gobierno de la Iglesia, así como asuntos relativos al culto. ${ }^{31}$

En este tablero conflictivo, el objetivo de Leibniz era alcanzar una formulación precisa y una resolución racional (filosófica) de las disputas doctrinarias, mostrando que existía, entre las diversas facciones, una compatibilidad al nivel de las creencias fundamentales. Este objetivo, sin embargo, no implicaba una reducción de las distintas confesiones a un dogma unificado, sino que apuntaba a un ecumenismo de convergencia, conservando una convivencia de las partes basada en lo que cada una tuviera de válido para alcanzar la verdad universal, aun cuando esto significara una devaluación del contenido dogmático. ${ }^{32}$ Por ello, la divisa de Leibniz para la reunión era "salva principiis partium". ${ }^{33}$

Esta tarea de negociación obligó a Leibniz a desplegar una actitud filosófica distinta: no se trataba de refutar (como en el caso del naturalismo), sino más bien de conciliar. Ella implicaba, a sus ojos, una estrategia que conjugaba dos elementos:

a. Un principio-marco que la filosofía debía demostrar, según el cual la verdad revelada no se opone a la verdad natural, ya que una verdad no puede contradecir a otra verdad. ${ }^{34}$ Este principio permite admitir que hay verdad en todas las partes, herederas de una misma revelación, y constituye un pilar fundamental para sustentar una irrenunciable aspiración filosófica leibniziana: la de consagrar el acuerdo entre la razón y la fe, estableciendo

\footnotetext{
31 Sleigh (1990), p.21-22.

32 Salas, p.406-409.

${ }^{33}$ AA I 6, p.380; Sleigh (1990), p.24.

${ }^{34} \mathrm{GP}$ VI, p.49 e 67-68, 84; Salas, p.411.
} 
las verdades racionales presentes en toda religión. Esta verdad compartida constituye la religión natural de la que habla el Leibniz de madurez. ${ }^{35}$

b. Una actitud más bien pragmática, ${ }^{36}$ plasmada en un conjunto de acciones y de recursos discursivos destinados a facilitar el diálogo y a lograr el acuerdo. Entre esos recursos se encuentra, por ejemplo, el empleo de géneros literarios menos confrontativos, como el diálogo, que escenifica la discusión entre voces divergentes y el arribo final a una respuesta aceptada por los interlocutores. ${ }^{37}$ Un recurso similar puede verse en el comentario "modesto" a escritos sobre cuestiones teológicas que eran debatidos en el contexto mismo de las negociaciones irénicas. ${ }^{38}$

Esta actitud conduce al Leibniz maduro a preferir la discusión amigable y privada sobre los puntos de controversia, antes que la disputa pública y escrita. Como señala Claire Rösler, la misma elaboración de la Théodicée se articula con el proyecto de alcanzar la paz entre las iglesias. ${ }^{39} \mathrm{Y}$ tal empresa requiere, para Leibniz, salir del terreno de las vanas chicanas. Así lo aclara en una carta a Jablonski del 26 de marzo de 1700:

$\mathrm{Y}$ en lugar de exponer actualmente a través de libros mis pensamientos sobre la Teodicea y las conexiones del mundo, dando a algunos espíritus pendencieros materia de vanas chicanas, yo estoy más bien inclinado a discutir amigablemente y a título privado sobre ellos con los excelentes teólogos de uno y otro partido, y solamente después, una vez que yo les haya

35 Salas, p.411, la carta a Sofía de abril de 1709.

${ }^{36}$ El término "pragmático" se emplea aquí en su sentido más habitual, que refiere a la preferencia por lo útil. No se emplea para aludir a un nivel de análisis del lenguaje (centrado en el uso del mismo y en su relación con los hablantes), ni mucho menos para referir a una corriente filosófica (el denominado "pragmatismo", bautizado como tal por Charles S. Peirce en el siglo XIX).

37 Un ejemplo temprano se encuentra en la Confessio philosophi de 1672-1673. En la Théodicée, Leibniz emplea un recurso algo similar, que es la estrategia irénica de "la medalla", donde una cara es una posición errónea y la contracara es su corrección por vía de demostración. Cf. GP VI, p.106.

38 Un claro ejemplo puede encontrarse en dos escritos que Leibniz redacta entre 1698 y 1699 , en respuesta a un texto de Daniel Ernst Jablonski (titulado "Kurtze Vorstellung") destinado a la negociación irénica entre las cortes de Hanover y de Berlín: el Tentamen expositionis irenicae trium potissimarum inter protestantes controversiarum y la Unvorgreiffliches Bedencken über eine Schrift genandt Kurtze Vorstellung. Claire Rösler considera que estos modestos ensayos anticipan y anuncian las tesis de la Théodicée de 1710. Cf. Rösler (2011), p. 287-290.

${ }^{39}$ Cf. Rösler (2011), p.286. 


\section{Dossiê Leibniz, Dissertatio - Volume Suplementar 03 UFPel [2016]}

dado satisfacción y ellos a mí, a hacerlos aparecer para dar servicio igualmente a los otros, si Dios me da vida (AA I, xviii, p.485).

¿Cómo se concilia esta búsqueda pragmática de acuerdo entre concepciones divergentes con la idea de que hay una filosofía verdadera? Esta tensión se acentúa si se tiene en cuenta que, en sus negociaciones irénicas, Leibniz sostiene un principio epistémico según el cual sólo se puede creer en algo si se cuenta con pruebas o fundamentos para ello.

En este punto, la posición de Leibniz parece ser la siguiente: los desacuerdos entre las partes son sobre aspectos que no hacen al núcleo esencial de la religión natural, por lo cual se puede, en definitiva, pertenecer a una misma iglesia sin tener pleno acuerdo en ellos. ${ }^{40} \mathrm{El}$ contenido de esa religión natural sería, pues, un contenido minimo, pero suficiente, para fundar (en términos epistémicos) la creencia, que conduce al amor a Dios. Y una religión consiste básicamente en la creencia y el culto. ${ }^{41} \mathrm{El}$ contenido de la religión natural parece estar centrado, para Leibniz, en la naturaleza de Dios (sus perfecciones), coincidiendo en parte o en todo con la teología natural. ${ }^{42}$

Si esto es correcto, parece ahora que, al menos en el terreno de las negociaciones irénicas, la cuestión de la predestinación (sobre la cual hay

\footnotetext{
${ }^{40}$ Así lo sugiere Leibniz, por ejemplo, en relación a la cuestión del decreto de elección y de reprobación, que constituía el principal desacuerdo doctrinal entre reformados y luteranos. En el Tentamen expositionis irenicae trium potissimarum inter protestantes convroversiarum, Leibniz afirma que no constituye un desacuerdo fundamental, y que puede ser resuelto fácilmente por medio de clarificaciones terminológicas, o bien puede ser tolerado. Cf. Rösler (2011), p.290.

${ }^{41}$ Salas, p.486.

42 GP VI 220, § 177.
} 
desacuerdo) no sería tan importante como Leibniz siempre la juzgó en el terreno de la verdadera filosofía. Ello acentúa la tensión entre el nivel pragmático (la búsqueda de consensos para unificar una base doctrinaria) y el práctico (los fundamentos racionales necesarios para establecer la virtud y la piedad cristiana).

En algunas de sus cartas a sus corresponsales intermediarios, Leibniz manifiesta su frustración frente a la intransigencia y al espiritu sectario de quienes se aferran a su adhesión confesional. ${ }^{43}$ Este espíritu es el nudo que Leibniz no logró desatar, la verdadera traba a la unión de los cristianos. Los intransigentes "desfiguran" a Dios, haciendo de él un ser afecto al culto exterior y vacío, que goza en ser incomprendido o que es cruel al extremo de condenar a los no iniciados en la fe. ${ }^{44}$

Aquí adquiere relevancia un concepto central, a mi juicio, de la filosofía práctica leibniziana: la verdadera piedad. Leibniz redacta varios escritos dedicados a ella, ${ }^{45}$ y ya desde sus notas de juventud define a la justicia universal como "pietas". ${ }^{46}$ La verdadera piedad es el amor a Dios, pero no un amor irracional, sino un amor basado en el conocimiento de sus perfecciones (su esencia). ${ }^{47}$ Para Leibniz, el signo más claro de esta virtud es el amor al prójimo, porque el conocimiento de Dios (el ser más perfecto) nos lleva a amarlo y a tratar de imitarlo, buscando realizar el bien común, en tanto constituye la mayor perfección

${ }^{43}$ AA I 8 125; Salas, pp.437-438 / Grua, pp.136-137; Salas, pp.411-415; Salas, pp.543-545 / Grua, p.205. ${ }^{44}$ Salas, pp.411-415.

${ }^{45}$ AA VI 4 1357-1366; Grua, pp.499-500.

${ }^{46} \mathrm{AA}$ VI 1, p.51, sus notas a la Philosophia practica de Thomasius.

${ }^{47} \mathrm{GP}$ VI, pp.27-28. 


\section{Dossiê Leibniz, Dissertatio - Volume Suplementar 03 I UFPel [2016]}

que puede alcanzarse en el mundo. ${ }^{48}$ Así, la verdadera piedad adquiere un estatuto preciado y una importancia vital en el pensamiento ético del Leibniz maduro, en tanto parece constituir una virtud por sí suficiente para elevar al espíritu del individuo por encima de toda división confesional.

Esta piedad sincera es la que Leibniz opone al sectarismo. ${ }^{49}$ Ella refleja su convicción, ingenua tal vez, en torno al poder transformador del conocimiento y de la verdad (de la filosofía) sobre el espíritu. Por ello, no es casual que su ideal político consista en promover el desarrollo del conocimiento, "hacer que florezca el imperio de la razón", ${ }^{50}$ tarea a la cual dedicó grandes esfuerzos en vida.

\section{Conclusiones: hacia un horizonte de convergencia}

Reconstruir la filosofía práctica de Leibniz es una tarea vasta, que nos enfrenta al desafío de interpretar la significación integral de su filosofía, y que requiere del abordaje de diversos elementos de su sistema. Algunos de esos elementos son, por ejemplo, el concepto de necesidad moral, o también los esbozos de una lógica deóntica que Leibniz formuló en su juventud, tomando como paradigma las nociones modales aléticas para definir conceptos jurídicos (los “modalia juris") y deducir teoremas a partir de ellos. ${ }^{51}$ El presente trabajo intenta

\footnotetext{
${ }^{48}$ Salas, p.425 / Grua, p.581.

${ }^{49}$ Salas, p.543 / Grua, p.205.

${ }^{50} \mathrm{GP}$ III, p.277.

${ }^{51} \mathrm{AA}$ VI 1, pp.459-480. Para un valioso análisis de este esbozo leibniziano de un sistema deductivo basado en los juris modalia, cf. Esquisabel (2011).
} 
solamente aportar algunas ideas matrices para encarar dicha tarea, desde una perspectiva que permita integrar y vincular distintos niveles de análisis.

En este sentido, la primera conclusión que puede formularse es que una reconstrucción sistemática y global de la filosofía práctica de Leibniz no puede reducirse a la ética y la jurisprudencia, sino que debe partir del reconocimiento de la importancia y la significación de orden práctico que poseen doctrinas capitales de su metafísica (como las teorías de la contingencia y de la libertad, la reflexión sobre la esencia del Ens perfectissimum y la explicación del origen del mal), así como los fundamentos de su física (como la explicación de la naturaleza de las fuerzas y la concepción de la naturaleza de los seres vivientes, que involucra doctrinas como las de la preformación y el desenvolvimiento-envolvimiento del animal preformado). ${ }^{52}$ En suma, esa filosofía práctica debe buscarse también en las porciones nodales de la propia filosofía teórica de Leibniz. El orden de prioridades de las cuestiones filosóficas (que tiene en su cima las cuestiones de la predestinación y la libertad, de la existencia de Dios y de la justicia divina frente a la existencia del mal) refleja, en definitiva, esta dimensión práctica.

Si esto es correcto, puede afirmarse que la filosofía en su conjunto adquiere, para Leibniz, una dimensión polemo-lógica: ella es el territorio primordial en el que tiene lugar la lucha por el establecimiento de la piedad. Ello no significa que Leibniz pretenda reducir la filosofía a un dispositivo retórico o dialéctico,

\footnotetext{
52 Las mencionadas doctrinas referidas a los seres vivientes constituyen hipótesis con apoyo experimental que se concilian armónicamente con una tesis metafísica vital para la práctica, a saber, aquella según la cual las sustancias no pueden nacer sino por creación ni perecer sino por aniquilación. Asimismo, tales doctrinas suponen un Dios infinitamente sabio como autor del universo.
} 


\section{Dossiê Leibniz, Dissertatio - Volume Suplementar 03 I UFPel [2016]}

ya que el filósofo está obligado a ceñirse a las reglas del arte de disputar. Más bien, ello implica reconocer que la filosofía es una tarea inevitablemente comprometida, a la vez, con el esclarecimiento y contra la tergiversación de las verdades que están al alcance de la razón. Por ello puede decirse que el principio de la concordancia entre fe y razón, junto con la tarea de su demostración, adquiere un papel fundacional en relación a la orientación general de la filosofía leibniziana, la cual parece ser, en su culminación y en su significación más profunda, una filosofía práctica. Toda filosofía es, en este sentido, práctica. Ello hace más necesaria la tarea de apuntalar la verdadera piedad.

Leibniz parece haber asumido de este modo su misión, tras leer a Lutero y a Valla. Además, la tarea práctica de la filosofía no es sólo clarificar cuál es el sumo bien, sino también realizarlo. Ello se debe a que la filosofía parece ser la única vía para alcanzar la verdadera piedad, ya que el conocimiento de Dios es la condición fundamental para amarlo de modo sincero. Y el amor a Dios, a su vez, constituye la razón más sólida para adquirir el hábito de amar a todos desinteresadamente (la justicia) y de obrar por el bien común. La ética de Leibniz es, pues, decididamente intelectualista: el conocimiento conduce a adquirir la virtud, en la medida en que conduce inevitablemente a Dios.

Un problema importante que sigue abierto es el de esclarecer cuáles serían los principios de ese orden moral universal que Leibniz parece postular, cuando concibe a la moral y a la jurisprudencia como ciencias basadas en verdades eternas. ¿Se identifican tales principios con los tres preceptos del derecho que Leibniz 
siempre aceptó? A mi juicio, el Leibniz maduro parece priorizar, por encima de toda tabla de preceptos, el amor sincero a Dios, como si se tratase de una condición suficiente para encauzar la acción acorde a la justicia. Así lo sugiere en una carta a Thomas Burnett, en la que afirma que la moral "demanda más de práctica que de preceptos". ${ }^{53}$ Sin embargo, como se vio, ese amor sincero recibe un aporte valioso del conocimiento filosófico. Como lo expresa el propio Leibniz en los NE, "yo he aprendido cada vez más cómo la moral recibe consolidación de los principios sólidos de la verdadera filosofía". ${ }^{54}$

Pero entonces, ¿cómo puede ser esta verdad, que es base de la piedad, objeto de negociación? En otras palabras, ¿cómo se concilia esta postulación de una verdad universal con una metodología pragmática para arribar a su formulación? Persiste, en Leibniz, esta tensión entre dos formas de racionalidad práctica distintas, una regida por la virtud y otra de carácter estratégico, ambas orientadas al mismo fin (el bien general). En la interpretación que pretendo tan sólo esbozar aquí, parece haber un horizonte de convergencia para esta tensión, y para todas las divisiones que Leibniz enfrentó en las disputas por la verdad y/o por la reunión de las iglesias. Ese horizonte se encuentra en el progreso de la razón. Leibniz reitera con gran convicción que lo mejor que puede hacerse para el bien común es fomentar el conocimiento de las ciencias,${ }^{55}$ porque ello hace más perfectos a los hombres y "equivale a hacer progresar el bien general". ${ }^{56}$ El conocimiento

${ }^{53}$ GP III, p.302. Agradezco a Paul Rateau por haberme hecho notar la importancia de este pasaje.

${ }^{54}$ AA VI 6, p.71.

${ }^{55}$ Cf. Grua, p.587.

${ }^{56}$ Cf. Salas, p.218 (carta Johann Friedrich del 29 de marzo de 1679, AA I 2, p.153 y ss.). 


\section{Dossiê Leibniz, Dissertatio - Volume Suplementar 03 I UFPel [2016]}

de las ciencias conduce, a su juicio, al conocimiento de Dios, lo cual ha de redundar en el amor a Dios y en la vocación práctica, a la que Leibniz ve como verdadero signo de la piedad.

Esta transformación del espíritu a través del conocimiento permite vislumbrar uno de los puntos más atractivos de la filosofía práctica leibniziana. Para concluir, cabe retomar, en relación a este punto, un concepto poco aprovechado que Leibniz formula en los Elementa verae pietatis. Allí distingue entre dos clases de "cogitatio": una cogitatio simplice ("imaginationem", "repraesentationem") y una cogitatio "cum agendi conatu", un pensamiento acompañado con el esfuerzo para actuar, al cual denomina "cogitatio practica" o "sententia". La filosofía parece corresponder, para Leibniz, a esta clase segunda clase de conocimiento. Para ella podría valer lo que dice Leibniz en el mismo pasaje: "es manifiesto que la fe (esto es, la sentencia), sin obras (esto es, sin caridad o esfuerzo de hacer el bien), no puede existir". ${ }^{57}$

\section{Ediciones de las obras de Leibniz}

LEIBNIZ, G.W. Sämtliche Schriften und Briefe, herausgegeben von der Deutschen Akademie der Wissenschaften, Darmstadt, 1923; Leipzig, 1938; Berlin, 1950 y prosigue.

${ }^{57} \mathrm{Cf} . \mathrm{AA}$ VI 4, p. 1361. 
Die philosophischen Schriften. GERHARDT, C. I. (ed.). 7 Bände,

Berlin 1875-1890.

. Textes inédits d'après les manuscrits de la bibliothèque provinciale de

Hanovre. GRUA, G. (ed.). 2 vols.. Paris: Presses Universitaires de France, 1948.

. Briefwechsel zwischen Leibniz und Christian Wolf. GERHARDT,

C. I. (ed.). Halle: 1860 (citado como LW).

. Mittheilungen aus Leibnizens ungedructen Scbriften. MOLLAT, G.

(ed). Leipzig: 1893 (citado como Mollat).

. Logico-Philosophical Puzzles in the Law, Artosi, Alberto, Pieri,

Bernardo y Sartor, Giovanni (eds.). Dordrecht-Heidelberg-Nueva YorkLondres: Springer, 2013.

- Escritos filosóficos. OLASO, E. (compilación, traducción y notas) e ZWANCK, T. (trad.). Buenos Aires: Charcas, 1982. (citado como de Olaso).

. Escritos de filosofía jurídica y politica. De SALAS ORTUETA, J.

(compilación y traducción). Madrid: Editora Nacional. 1984. (citado como Salas).

\section{REFERENCIAS BIBLIOGRAFICAS}

ADAMS, Robert Merrihew. Leibniz. Determinist, Theist, Idealist. New York: Oxford University Press: 1994. 
. "Moral Necessity". En RUTHERFORD y

COVER (ed.). Leibni₹. Nature and Freedom Oxford: Oxford University Press, pp. 181-193, 2005.

ANFRAY, Jean-Pascal. "Leibniz, le choix du meilleur et la nécessité morale". En RATEAU, Paul (ed.). Lectures et interprétations des Essais de théodicée de G. W. Leibniz, Studia Leibnitiana - Sonderhefte 40. Stuttgart, Franz Steinter Verlag, 2011, pp.59-78.

BEGBY, Endre. "Leibniz on Determinism and Divine Foreknowledge". Studia Leibnitiana 37, 2005, pp.83-98.

BLUMENFELD, David. "Leibniz`s Theory of the Striving Possibles". Studia Leibnitiana 5, 1973, pp.163-177.

BROWN, Stuart. Leibniz: Minneapolis: University of Minnesota Press, 1984.

COUTURAT, Louis (1961). La logique de Leibniz. Reimpresión Hildesheim: Georg Olms, 1988.

. “On Leibniz's Metaphysics”, en Frankfurt, H. G. (ed.), Leibniz: A Collection of Critical Essays. Garden City, New York: Anchor Books: 19-45.

DAVIDSON, Jack D. "Video meliora proboque, deteriora sequor. Leibniz on the Intellectual Source of Sin". En: En RUTHERFORD y COVER (ed.). 
Leibniz: Nature and Freedom Oxford: Oxford University Press, pp.234-253, 2005.

ESCOBAR VIRÉ, Maximiliano. "El problema del continuum y sus implicancias en la teoría leibniciana de la sustancia". Revista de Filosofía y Teoría Politica 38, 2007, pp.67-93.

. "El argumento ontológico, la necesidad absoluta y el problema de la contingencia en Leibniz". Revista Latinoamericana de Filosofía, Vol. XXXVII, No 1, 2011, pp.97-125.

. "Legitimidad y piedad en Leibniz: el orden civil al servicio del progreso racional”. En: Lenguaje, voluntad e igualdad en la sociedad moderna: homenaje a Jean-Jaques Rousseau: Actas III Jornadas Nacionales de Filosofía Moderna. PULLEY, R. y MARTÍNEZ SAEZ, N. (comp.). Mar del Plata: Universidad Nacional de Mar del Plata, 2012, pp.185193.

ESQUISABEL, Oscar. "El tratamiento de las modalidades aléticas y deónticas en Leibniz". En: LEGIRS, J. (comp.). Documentos del CIECE 7, 2011, PP.7-23.

FRIEDMANN, Georges. Leibniz et Spinoza. Paris: Gallimard, 1946. 


\section{Dossiê Leibniz, Dissertatio - Volume Suplementar 03 I UFPel [2016]}

GRUA, Gaston. Jurisprudence universelle et Théodicée selon Leibniæ. Paris. Presses Universitaires de France, 1953.

HACKING, Ian. "Infinite Analysis". Studia Leibnitiana 6, 1974, pp.126-130. . “A Leibnizian Theory of Truth”. En HOOKER, M. (ed.).,

Leibniz: Critical and Interpretive Essays. Minneapolis: University of Minnesota Press, pp.185-195, 1982.

HOSTLER, John. "Some remarks on `omne possibile exigit existire"”. Studia Leibnitiana 5, 1973, pp.281-285. . Leibniz's Moral Pbilosophy. Harper \& Row Publishers, 1975.

JOLLEY, Nicholas (ed). The Cambridge Companion to Leibni;. Cambridge: Cambridge University Press, 1995.

KRAYE, Jill. “Conceptions of Moral Philosophi”. En: GARBER, Daniel y AYERS, Michael (ed.). The Cambridge History of Seventeenth-Century Pbilosophy. Cambridge: Cambridge University Press, vol II, pp.1279-1316, 2008.

LOVEJOY, Arthur Onken. "Plenitude and Sufficient Reason in Leibniz and Spinoza". En FRANKFURT, H. G. (ed.). Leibniæ: A Collection of Critical Essays. Garden City, New York: Anchor Books, pp.281-334,1972. 
MOLL, Konrad (1999), "Deus sive harmonia universalis est ultima ratio rerum: the conception of God in Leibniz's early philosophy", en Bown, Stuart (ed.), The Young Leibniz and his Philosophy (1646-76). Dordrecht: Kluwer Academic Publishers: pp. 65-78.

MONDADORI, Fabrizio. "Reference, Essentialism and Modality in Leibniz's Metaphysics". Studia Leibnitiana 5, 1973, pp.74-101. . "Necessity ex Hypothesi”. En: Centro Fiorentino,

The Leibniz Renaissance, pp.191-222,1986.

MURRAY, Michael J. "Leibniz on Divine Foreknowledge of Future Contingents and Human Freedom". Philosophy and Phenomenological Research 55, 1995, pp75-108.

. "Intellect, Will and Freedom: Leibniz and his Precursors". Leibniz Review 6, 1996, pp.25-59.

PASINI, Enrico. “Complete Concepts as Histories”. Studia Leibnitiana 42, 2012, pp.229-243.

PIRO, Francesco. "Leibniz and ethics: the years 1669-72". En: BROWN, Stuart (ed.). The Young Leibniz and his Philosophy (1646-76). Dordrecht: Kluwer Academic Publishers, pp.147-167, 1999. 


\section{Dossiê Leibniz, Dissertatio - Volume Suplementar 03 I UFPel [2016]}

. "For a History of Leibniz's Principle of Sufficient

Reason. First Formulations and their Historical Background”, en DASCAL, Marcelo (ed.). Leibniæ: What Kind of Rationalist?. Tel Aviv: Springer, pp.463478, 2008.

POSER, Hans. Zur Theorie der Modalbegriffe bei G.W. Leibniz. Studia Leibnitiana Supplementa VI, 1969. Wiesbaden: Franz Steiner Vg.

RATEAU, Paul. La question du mal chez Leibniæ. Fondaments et élaboration de la Théodicée. Paris: Honoré Champions Éditeur, 2008.

RESCHER, Nicholas. "Contingence in the Philosophy of Leibniz". The Philosophical Review 61, 1952, pp. 26-39. The Philosophy of Leibniæ. Prentice-Hall, Englewood

Cliffs, 1967.

. Leibniz's Metaphysics of Nature. Dordrecht: D. Reidel

Publishing Company, 1981.

"Contingentia Mundi. Leibniz on the World`s Contingency". Studia Leibnitiana 33, 2001, pp.145-162.

"Leibniz on God`s Free Will and the World`s Contingency". Studia Leibnitiana 34, 2002, pp.208-220. 
"On Some Purported Obstacles to Leibniz's Optimalism”. Studia Leibnitiana 37, 2005, pp.131-146.

RÖSLER, Claire. "L'influence sur la Théodicée du negotium irenicum entrepris par G. W. Leibniz et D. E. Jablonski”. En: Rateau, Paul (ed.), Lectures et interprétations des Essais de théodicée de G. W. Leibniz. Studia Leibnitiana Sonderhefte 40. Stuttgart: Franz Steiner Verlag, 2011, pp.285-306.

RUSSELL, Bertrand. Exposición crítica de la filosofía de Leibniz. Buenos Aires: Siglo veinte, 1977 .

RUTHERFORD, Donald. Leibniz and the Rational Order of Nature. Cambridge: Cambridge University Press, 1995.

, D. \& COVER, J. A. (ed.). Leibniæ. Nature and Freedom. New York: Oxford University Press, 2005.

SAAME, Otto. El principio de razón en Leibniæ. Barcelona: Laia, 1987.

SCHEPERS, Heinrich. "Posibilidad y contingencia. Historia de la terminología filosófica anterior a Leibniz". Revista de Filosofía y Teoría Política 33, 1999, pp.43-63.

SLEIGH, Robert C. (Jr.) "Truth and Sufficient Reason in the Philosophy of Leibniz". En HOOKER, M. (ed.). Leibniæ: Critical and Interpretative Essays. Minneapolis: University of Minnesota Press, pp. 209-42, 1982. 
Dossiê Leibniz, Dissertatio - Volume Suplementar 03 I UFPel [2016]

- Leibniz \& Amauld. A Commentary on Their

Correspondence. New Haven/London: Yale University Press, 1990.

E-mail: m_escobarvire@yahoo.com 